\title{
Erratum: Two-Stage Dynamics of In Vivo Bacteriophage Genome Ejection [Phys. Rev. X 8, 021029 (2018)]
}

Yi-Ju Chen, David Wu, William Gelbart, Charles M. Knobler, Rob Phillips, and Willem K. Kegel

(-) (Received 1 October 2019; accepted 20 December 2019; published 6 February 2020)

DOI: $10.1103 /$ PhysRevX.10.019901

We missed a factor in the definition of the subdiffusion coefficient $D$ in Eq. (6) that takes into account the proper dimension of $D$, which should be in the units $\mathrm{m}^{2} / \mathrm{s}^{\alpha}$. Pragmatically, we fix that by absorbing a factor on the order of 1 with the appropriate unit into the quantity $f$ in the definition of $D$ below Eq. (6). Therefore, $f$ should have the unit s ${ }^{\alpha-1}$ rather than being a number. In other words, the value of $f$ in line 8 below Eq. (6) and line 7 below Eq. (8) should be " $f \approx 10 \mathrm{~s}^{\alpha-1}$." As a consequence, $\chi$ as defined above Eq. (8) has the unit $\mathrm{s}^{-\alpha}$. This correction has no impact on the conclusions of the paper.

Published by the American Physical Society under the terms of the Creative Commons Attribution 4.0 International license. Further distribution of this work must maintain attribution to the author(s) and the published articles title, journal citation, and DOI. 